\title{
Site Mixing for Engineering Magnetic Topological Insulators
}

\author{
Yaohua Liu $\odot,{ }^{1, *}$ Lin-Lin Wang, ${ }^{2}$ Qiang Zheng, ${ }^{3}$ Zengle Huang, ${ }^{4}$ Xiaoping Wang $\odot,{ }^{1}$ Miaofang Chi, ${ }^{5}$ Yan Wu๑, \\ Bryan C. Chakoumakos $\odot,{ }^{1}$ Michael A. McGuire $\odot,{ }^{5}$ Brian C. Sales $\odot,{ }^{5}$ Weida Wu $\odot,{ }^{4}$ and Jiaqiang Yan $\odot^{5,3, \dagger}$ \\ ${ }^{1}$ Neutron Scattering Division, Oak Ridge National Laboratory, Oak Ridge, Tennessee 37831, USA \\ ${ }^{2}$ Division of Materials Science and Engineering, Ames Laboratory, Ames, Iowa 50011, USA \\ ${ }^{3}$ Department of Materials Science and Engineering, University of Tennessee, \\ Knoxville, Tennessee 37996, USA \\ ${ }^{4}$ Department of Physics and Astronomy, Rutgers University, Piscataway, New Jersey 08854, USA \\ ${ }^{5}$ Materials Science and Technology Division, Oak Ridge National Laboratory, \\ Oak Ridge, Tennessee 37831, USA
}

(Received 1 September 2020; revised 9 December 2020; accepted 15 March 2021; published 12 May 2021)

\begin{abstract}
The van der Waals compound, $\mathrm{MnBi}_{2} \mathrm{Te}_{4}$, is the first intrinsic magnetic topological insulator, providing a materials platform for exploring exotic quantum phenomena such as the axion insulator state and the quantum anomalous Hall effect. However, intrinsic structural imperfections lead to bulk conductivity, and the roles of magnetic defects are still unknown. With higher concentrations of the same types of magnetic defects, the isostructural compound $\mathrm{MnSb}_{2} \mathrm{Te}_{4}$ is a better model system for a systematic investigation of the connections among magnetism, topology, and lattice defects. In this work, the impact of antisite defects on the magnetism and electronic structure is studied in $\mathrm{MnSb}_{2} \mathrm{Te}_{4} . \mathrm{Mn}-\mathrm{Sb}$ site mixing leads to complex magnetic structures and tunes the interlayer magnetic coupling between antiferromagnetic and ferromagnetic. The detailed nonstoichiometry and site mixing of $\mathrm{MnSb}_{2} \mathrm{Te}_{4}$ crystals depend on the growth parameters, which can lead to $\approx 40 \%$ of $\mathrm{Mn}$ sites occupied by $\mathrm{Sb}$ and $\approx 15 \%$ of $\mathrm{Sb}$ sites by $\mathrm{Mn}$ in as-grown crystals. Single-crystal neutron diffraction and electron microscopy studies show nearly random distribution of the antisite defects. Band structure calculations suggest that the $\mathrm{Mn}-\mathrm{Sb}$ site mixing favors a ferromagnetic interlayer coupling, consistent with experimental observation, but is detrimental to the band inversion required for a nontrivial topology. Our results suggest a long-range magnetic order of $\mathrm{Mn}$ ions sitting on $\mathrm{Bi}$ sites in $\mathrm{MnBi}_{2} \mathrm{Te}_{4}$. The effects of site mixing should be considered in all layered heterostructures that consist of alternating magnetic and topological layers, including the entire family of $\mathrm{MnTe}\left(\mathrm{Bi}_{2} \mathrm{Te}_{3}\right)_{n}$, its $\mathrm{Sb}$ analogs, and their solid solution.
\end{abstract}

DOI: 10.1103/PhysRevX.11.021033

\section{INTRODUCTION}

Structural imperfection in crystalline materials can have profound effects on the electronic, magnetic, optical, mechanical, and thermal properties. For topological insulators, the surface state is protected against nonmagnetic lattice defects due to spin-momentum locking. Magnetic dopants have been purposely introduced to topological insulators to induce magnetism, which breaks time-reversal symmetry. This approach led to the first experimental observation of the quantum anomalous Hall effect

\footnotetext{
*liuyh@ornl.gov

†yanj@ornl.gov
}

Published by the American Physical Society under the terms of the Creative Commons Attribution 4.0 International license. Further distribution of this work must maintain attribution to the author(s) and the published article's title, journal citation, and DOI.
Subject Areas: Condensed Matter Physics, Magnetism, Topological Insulators
(QAHE) in $\mathrm{Cr}_{0.15}\left(\mathrm{Bi}_{0.1} \mathrm{Sb}_{0.9}\right)_{1.85} \mathrm{Te}_{3}$ films [1]. This complex chemical formula highlights the importance of identification and fine manipulation of lattice defects in realizing the exotic properties of topological materials. Because of the random distribution of $\mathrm{Cr}$ in the $\mathrm{Bi}$ layer, however, the QAHE was only observed at extremely low temperatures of $30 \mathrm{mK}$, even though the onset of magnetic ordering occurs at $\approx 15 \mathrm{~K}$ [1].

$\mathrm{MnBi}_{2} \mathrm{Te}_{4}$, a natural heterostructure of magnetic (MnTe) and topological $\left(\mathrm{Bi}_{2} \mathrm{Te}_{3}\right)$ ingredients, in principle should be an ideal platform for observing the QAHE and axion behavior since it is a stoichiometric compound instead of a random alloy. However, $\mathrm{MnBi}_{2} \mathrm{Te}_{4}$, like any crystalline compound, will have defects. As shown in Fig. 1(a), $\mathrm{MnBi}_{2} \mathrm{Te}_{4}$ consists of septuple layers of Te-Bi-Te-MnTe-Bi-Te stacked along the crystallographic $c$ axis [2]. Below $T_{N}=25 \mathrm{~K}$, the high spin $\mathrm{Mn}^{2+}\left(d^{5}, S=5 / 2\right)$ ions order ferromagentically in each septuple layer. The layers then couple antiferromagnetically with neighboring layers 

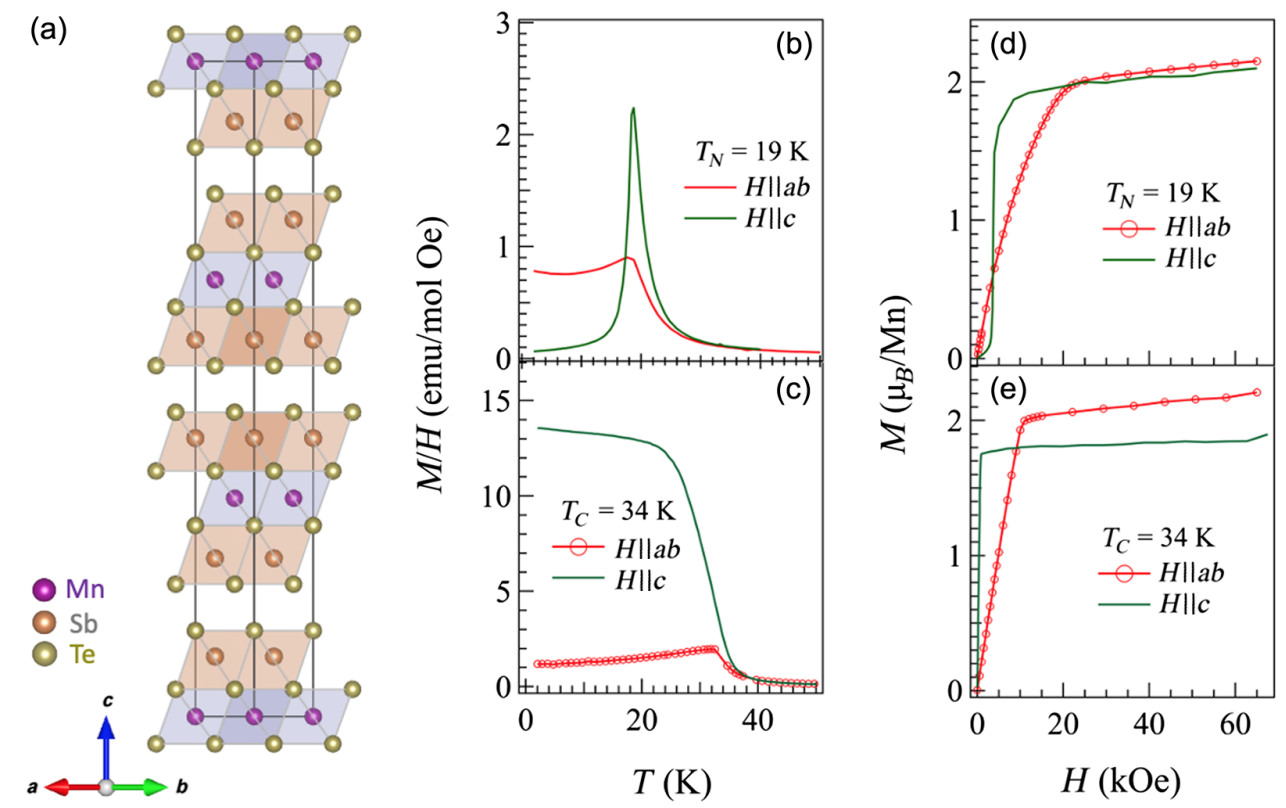

FIG. 1. Different growth temperatures result in distinct magnetic properties of $\mathrm{MnSb}_{2} \mathrm{Te}_{4}$ single crystals. (a) Ideal crystal structure of $\mathrm{MnSb}_{2} \mathrm{Te}_{4}$, isostructural to $\mathrm{MnBi}_{2} \mathrm{Te}_{4}$. (b),(c) Temperature dependence of magnetic susceptibility measured in a field-cooled mode with an applied magnetic field of $1 \mathrm{kOe}$. (d),(e) Field dependence of magnetization at $2 \mathrm{~K}$. The applied magnetic field is either along $(H \| c)$ or perpendicular to $(H \| a b)$ the crystallographic $c$ axis. The crystal with $T_{N}=19 \mathrm{~K}$ was grown at $620^{\circ} \mathrm{C}$, while the one with $T_{C}=34 \mathrm{~K}$ was grown at $640^{\circ} \mathrm{C}$.

forming the so-called A-type antiferromagnetic (AFM) structure [3,4]. Therefore, in addition to those lattice defects observed in $\mathrm{Bi}_{2} \mathrm{Te}_{3}$ [5-10], magnetic defects are expected in $\mathrm{MnBi}_{2} \mathrm{Te}_{4}$. The question then becomes, what type and what concentration of defects can be tolerated to preserve the entangled quantum ground state? How do these defects impact the magnetism and the observation of the QAHE? These questions are very general and apply to any material proposed for observation of the QAHE.

Experimental investigations [4,11-14] reported antisite disorders of $\mathrm{Mn}_{\mathrm{Bi}}^{\prime}(\mathrm{Mn}$ at the $\mathrm{Bi}$ site with one negative charge following Kroger-Vink notation), $\mathrm{Bi}_{\mathrm{Mn}}$ ( $\mathrm{Bi}$ at $\mathrm{Mn}$ site with a positive charge), $\mathrm{Bi}_{\mathrm{Te}}^{\prime}$, and likely $\mathrm{Mn}$ vacancies in $\mathrm{MnBi}_{2} \mathrm{Te}_{4}$ crystals or films. Density functional theory (DFT) calculations [15] suggest the lattice mismatch between $\mathrm{MnTe}$ and $\mathrm{Bi}_{2} \mathrm{Te}_{3}$ sheets facilitates the formation of Mn-Bi site mixing. These lattice defects lead to the bulk conductivity and affect the spin wave spectra [16,17]. However, it is unknown whether the $\mathrm{Mn}$ ions at $\mathrm{Bi}$ site order magnetically, how the magnetic defects affect the magnetism, and whether they are related to the diverse surface states resolved by angle-resolved photoemission studies [3,13,18-23]. Furthermore, ample experimental evidence suggests these magnetic defects also exist in other $\operatorname{MnTe}\left(\mathrm{Bi}_{2} \mathrm{Te}_{3}\right)_{n}$ members with $n>1$ [24-32]. Therefore, it is critical to understand the formation, distribution, consequences, and manipulation of the magnetic defects in this family of compounds.

$\mathrm{MnSb}_{2} \mathrm{Te}_{4}$ is a model system for such a systematic investigation of the connections among magnetism, topology, and lattice defects. $\mathrm{MnSb}_{2} \mathrm{Te}_{4}$ is isostructural to $\mathrm{MnBi}_{2} \mathrm{Te}_{4}$, and these two compounds have the same types of defects [15]. However, the concentration of lattice defects in $\mathrm{MnBi}_{2} \mathrm{Te}_{4}$ is only a few percent [4], which is difficult to systematically study. In contrast, $\mathrm{MnSb}_{2} \mathrm{Te}_{4}$ tends to have higher concentrations of magnetic defects because the difference of ionic size and electronegativity between $\mathrm{Mn}$ and $\mathrm{Sb}$ is much smaller than that between $\mathrm{Mn}$ and $\mathrm{Bi}$ in $\mathrm{MnBi}_{2} \mathrm{Te}_{4}$. From a crystal growth point of view, $\mathrm{MnSb}_{2} \mathrm{Te}_{4}$ crystals can be grown easily in a wide temperature range which makes it possible to fine-tune the concentrations of lattice defects and hence the magnetism by varying the growth temperatures. The growth of $\mathrm{MnBi}_{2} \mathrm{Te}_{4}$ has been quite challenging and the crystallization occurs in a narrow temperature window of $\approx 10^{\circ} \mathrm{C}$ $[4,13]$. Finally, while $\mathrm{MnSb}_{2} \mathrm{Te}_{4}$ single crystals are reported to order antiferromagnetically [33], polycrystalline samples are reported to have ferrimagnetic septuple layers that are coupled ferromagnetically due to the presence of $\mathrm{Mn}$ at the $\mathrm{Sb}$ site [34]. It is unknown whether this complex magnetic structure is inherent to the polycrystalline sample or if the discrepancy between single-crystal and polycrystalline samples results from a different amount of magnetic defects and signals their essential roles. Of particular interest is the origin of the ferromagnetic (FM) interseptuple-layer coupling, which might be employed to fine-tune the magnetism of $\mathrm{MnBi}_{2} \mathrm{Te}_{4}$ for the observation of the QAHE at elevated temperatures.

In this work, we perform a systematic study of the magnetic defects in $\mathrm{MnSb}_{2} \mathrm{Te}_{4}$ single crystals with different 
magnetic ground states and tunable magnetic ordering temperatures. Our results show that the randomly distributed $\mathrm{Mn}-\mathrm{Sb}$ antisite defects favor a FM interlayer coupling but are detrimental to the band inversion necessary for a nontrivial band topology. Our results imply that $\mathrm{Mn}_{\mathrm{Bi}}^{\prime}$ ions in $\mathrm{MnBi}_{2} \mathrm{Te}_{4}$ order ferromagnetically below $T_{N}=25 \mathrm{~K}$, but they are antiferromagentically coupled to $\mathrm{Mn}_{\mathrm{Mn}}^{\times}$ions in each septuple layer. Partial substitution of Bi by magnetic ions in $\mathrm{MnBi}_{2} \mathrm{Te}_{4}$ might be a valid approach toward a FM interseptuple-layer coupling and quantum Hall effect at zero magnetic field.

\section{EXPERIMENTAL DETAILS}

$\mathrm{MnSb}_{2} \mathrm{Te}_{4}$ single crystals were grown out of a Sb-Te flux [4]. After homogenizing the starting materials at $900^{\circ} \mathrm{C}$ overnight, the mixture was furnace cooled to a certain temperature between $620^{\circ} \mathrm{C}$ and $640{ }^{\circ} \mathrm{C}$ and kept at the selected temperature for 2 weeks. The fixed-temperature growth was employed to obtain uniform crystals because the lattice defects are sensitive to the growth temperatures and starting compositions. The crystals were then separated from $\mathrm{Sb}-\mathrm{Te}$ flux by decanting. In this work, we focus on the crystals grown at three different temperatures of $620^{\circ} \mathrm{C}$, $630^{\circ} \mathrm{C}$, and $640^{\circ} \mathrm{C}$. The growth temperature determines the nonstoichiometry and site mixing and thus the magnetic order. We also tried crystal growths starting with a different ratio of $\mathrm{MnTe}: \mathrm{Sb}_{2} \mathrm{Te}_{3}$, which also affects the nonstoichiometry and site mixing in $\mathrm{MnSb}_{2} \mathrm{Te}_{4}$ and enables a finetuning of the magnetic ordering temperature up to $50 \mathrm{~K}$ (Fig. S4 in Supplemental Material [35]).

Elemental analysis on cleaved surfaces was performed using either the energy dispersive (EDS) or the wavelength dispersive (WDS) spectroscopy techniques. The EDS measurement was performed using a Hitachi TM-3000 tabletop electron microscope equipped with a Bruker Quantax 70 energy dispersive $\mathrm{x}$-ray system. The WDS measurement was performed using a JEOL JXA-8200X electron microprobe analyzer instrument equipped with five crystal-focusing spectrometers for wavelength dispersive x-ray spectroscopy. Magnetic properties were measured with a Quantum Design (QD) magnetic property measurement system in the temperature range $2.0 \leq T \leq 300 \mathrm{~K}$. The temperature- and fielddependent electrical resistivity data were collected using a QD physical property measurement system.

High-resolution single-crystal neutron diffraction data were collected at $300 \mathrm{~K}$ on TOPAZ at the Spallation Neutron Source (SNS) to determine the average nuclear structures. The integrated Bragg intensities are obtained using the 3D ellipsoidal $Q$-space integration method and are corrected for background using MANTID software [36]. Data reduction including Lorentz and absorption corrections as well as spectrum, detector efficiency, data scaling, and normalization are carried out with the ANVRED3 program [37]. The structure models were analyzed with the SHELX program [38]. Low-temperature single-crystal neutron diffraction experiments were carried out on beam line CORELLI [39] at SNS and the Four-Circle Diffractometer (HB3A) at the High Flux Isotope Reactor (HFIR) to determine the average magnetic structure. Possible magnetic structures were investigated by the representation analysis using the SARAh program [40], as well as the magnetic space group approach, where the maximal magnetic space groups were obtained from the MAXMAGN program [41]. Nuclear and magnetic structure refinements were carried out with the FullProf suite program [42]. Additionally, the temperature dependence of the magnetic order and potential diffuse scattering in all three samples were investigated on CORELLI.

Scanning transmission electron microscopy (STEM) observations were performed on a Nion UltraSTEM100, equipped with a cold field-emission gun and a corrector of third- and fifth-order aberrations, operated at the accelerating voltage of $100 \mathrm{kV}$. About $2 \times 2 \mu \mathrm{m}^{2}$ thin specimens of $\mathrm{MnSb}_{2} \mathrm{Te}_{4}$ were prepared by focused ion beam, and subsequently by ion milling at a low voltage of $1.5 \mathrm{kV}$ and liquid nitrogen temperature for $20 \mathrm{~min}$. High-angle annular dark-field (HAADF) STEM images were collected with a probe convergence angle of $30 \mathrm{mrad}$ and an inner collection angle of $86 \mathrm{mrad}$. Scanning tunneling microscopy (STM) measurements were performed at $48 \mathrm{~K}$ in an Omicron LT STM with base pressure $1 \times 10^{-10}$ mbar. Electrochemically etched tungsten tips were conditioned on a clean $\mathrm{Au}(111)$ surface before STM experiments. Single crystals of $\mathrm{MnSb}_{2} \mathrm{Te}_{4}$ were cleaved in situ at room temperature and immediately inserted into a cold STM head.

Band structures with spin-orbit coupling (SOC) in density functional theory $[43,44]$ have been calculated with the PBE [45] exchange-correlation functional, a plane-wave basis set and projected augmented wave method as implemented in VASP $[46,47]$. To account for the half-filled strongly localized Mn $3 d$ orbitals, a Hubbard-like $U$ [48] value of $3.0 \mathrm{eV}$ is used. The $(2 \times 2 \times 2) \mathrm{MnSb}_{2} \mathrm{Te}_{4}$ rhombohedral unit cell is sampled with a Monkhorst-Pack [49] $(6 \times 6 \times 3) k$-point mesh including the $\Gamma$ point and a kinetic energy cutoff of $270 \mathrm{eV}$. For band structure calculations, the experimental lattice parameters have been used with atoms fixed in their bulk positions. The supercell has also been fully relaxed in PBEsol [50] exchange-correlation functional with $U$ for total energy difference.

\section{RESULTS}

\section{A. Distinct magnetic properties}

Figures 1(b)-1(e) show two distinct types of magnetic properties of our $\mathrm{MnSb}_{2} \mathrm{Te}_{4}$ crystals. An AFM-like anisotropic magnetic susceptibility [see Fig. 1(b)], $\chi$, for the crystals grown at $620^{\circ} \mathrm{C}$ is in contrast to a FM-like temperature dependence [see Fig. 1(c)] for those grown at $640{ }^{\circ} \mathrm{C}$. The magnetic susceptibility for both batches shows a similar temperature dependence when the external 
magnetic field is applied perpendicular to the crystallographic $c$ axis. However, when the magnetic field is applied along the $c$ axis, $\chi_{c}$ in Fig. 1(b) decreases sharply when cooling below $19 \mathrm{~K}$, while $\chi_{c}$ in Fig. 1(c) shows a rapid increase upon cooling below the magnetic ordering temperature. The former is similar to that observed for $\mathrm{MnBi}_{2} \mathrm{Te}_{4}$ suggesting an AFM interlayer coupling [4,11-14,33]. The latter suggests a FM interlayer coupling, as previously observed in polycrystalline samples [34]. This is also supported by the field dependence of magnetization shown in Figs. 1(d) and 1(e). A well-defined spin-flip transition is observed in Fig. 1(d) when the field is applied along the $c$ axis. This spin-flip transition is absent in Fig. 1(e), also suggesting a FM interaction along the $c$ axis. When the magnetic field is applied perpendicular to the $c$ axis, a similar field dependence is observed for both crystals; however, the magnetic field at which the magnetization tends to saturate decreases from about $25 \mathrm{kOe}$ in Fig. 1(d) to $10 \mathrm{kOe}$ in Fig. 1(e). The saturation moment obtained by extrapolating the $M(H)$ curve to $H=0$ is 1.95(2) and $1.98(2) \mu_{B} / \mathrm{Mn}$, respectively. For both crystals, the anisotropic field and temperature dependencies of magnetization suggest the ordered magnetic moments are along the $c$ axis.

The magnetic measurements suggest that the sign of the interlayer interaction is tunable by the growth temperature. The crystals grown at $620^{\circ} \mathrm{C}$ show the AFM-like $\chi(T)$, as in Fig. 1(b), and those grown above $625^{\circ} \mathrm{C}$ show a FM-like one, as in Fig. 1(c). While all AFM-like crystals order magnetically at the same $T_{N}$, the magnetic ordering temperature of FM-like crystals can be tuned in the temperature range $24-50 \mathrm{~K}$ by controlling the growth parameters as shown in Fig. S4 in Supplemental Material [35]. As described below, the magnetic ordering temperature and saturation moment depend on the nonstoichiometry and distribution of $\mathrm{Mn}$. In the following, we refer to those three crystals that are thoroughly investigated as $\mathrm{T}_{N} 19 \mathrm{~K}, \mathrm{~T}_{C} 24 \mathrm{~K}$, and $\mathrm{T}_{C} 34 \mathrm{~K}$, respectively, with $\mathrm{T}_{N}$ indicating AFM-interlayer coupling, $\mathrm{T}_{C}$ for FM-interlayer coupling, and the number as the magnetic ordering temperature.

\section{B. Site mixing and magnetic structure from neutron single-crystal diffraction}

The anisotropic magnetic properties suggest that the interlayer coupling can be either FM or AFM although in both cases the ordered moment is aligned along the $c$ axis. To understand the different magnetic behaviors and determine the detailed magnetic structures, we performed singlecrystal neutron diffraction experiments in a wide temperature range using multiple diffractometers. High-resolution diffraction data collected at $300 \mathrm{~K}$ on beam line TOPAZ confirmed that the average structure can be well described by the space group $R \overline{3} m$ for both types of crystals (Fig. S1 in Supplemental Material [35]), similar to previous reports on powder [34] or single-crystal samples [33,51]. Neutron diffraction is sensitive to the $\mathrm{Mn}$-Sb mixing because of the high scattering contrast between $\mathrm{Mn}$ and $\mathrm{Sb}$ nuclei. Refinement of neutron diffraction data shows significant $\mathrm{Mn}-\mathrm{Sb}$ mixing at both $\mathrm{Mn}$ and $\mathrm{Sb}$ sites for all samples with about $13 \%-16 \% \mathrm{Mn}_{\mathrm{Sb}}^{\prime}$ and $32 \%-41 \% \mathrm{Sb}_{\mathrm{Mn}}^{\circ}$. The chemical formulas are $\left(\mathrm{Mn}_{0.588(2)} \mathrm{Sb}_{0.412(2)}\right)\left(\mathrm{Sb}_{0.871(2)} \mathrm{Mn}_{0.129(2)}\right)_{2} \mathrm{Te}_{4}$, $\left(\mathrm{Mn}_{0.635(2)} \mathrm{Sb}_{0.365(2)}\right)\left(\mathrm{Sb}_{0.850(2)} \mathrm{Mn}_{0.150(2)}\right)_{2} \mathrm{Te}_{4}, \quad$ and $\left(\mathrm{Mn}_{0.674(4)} \mathrm{Sb}_{0.326(4)}\right)\left(\mathrm{Sb}_{0.842(3)} \mathrm{Mn}_{0.158(3)}\right)_{2} \mathrm{Te}_{4}$ for $\mathrm{T}_{N} 19 \mathrm{~K}$, $\mathrm{T}_{C} 24 \mathrm{~K}, \mathrm{~T}_{C} 34 \mathrm{~K}$, respectively (Tables $\mathrm{S} 1-\mathrm{S} 4$ in Supplemental Material). The refined elemental ratio agrees with that obtained from elemental analyses as summarized in Table S6 in Supplemental Material. Interestingly, with increasing growth temperatures, $\mathrm{MnSb}_{2} \mathrm{Te}_{4}$ crystals contain more $\mathrm{Mn}$ ions on both $\mathrm{Mn}$ and $\mathrm{Sb}$ sites. This influences the competing magnetic interactions and hence the sign of interseptuple-layer coupling and the magnetic ordering temperature.

Low-temperature diffraction data were collected on CORELLI to investigate the magnetic structure and potential diffuse scattering. Datasets for refinement were collected for $\mathrm{T}_{N} 19 \mathrm{~K}$ and $\mathrm{T}_{C} 24 \mathrm{~K}$ at both 30 and $6.5 \mathrm{~K}$. The diffraction patterns collected above magnetic ordering temperature are similar for both crystals [see, e.g., Fig. S2(a) in Supplemental Material [35]]. However, they are quite different in the magnetically ordered state at $6.5 \mathrm{~K}$. The diffraction pattern in Fig. 2(a) for $T_{C} 24 \mathrm{~K}$ is similar to that collected at $30 \mathrm{~K}$, but the intensity of some peaks is enhanced, as highlighted by the white arrows (Fig. S2 in Supplemental Material), suggesting a magnetic wave vector of $k_{\mathrm{FM}}=(000) . \mathrm{T}_{N} 19 \mathrm{~K}$ [see Fig. 2(b)] shows a completely new set of peaks at the half- $L$ positions below $\mathrm{T}_{N}$, suggesting a magnetic wave vector of $k_{\mathrm{AFM}}=(001.5)$. Figure 2(c) shows the temperature dependence of intensities of selected Bragg peaks which confirms the magnetic ordering wave vectors as well as the magnetic ordering temperatures as found from magnetic and transport measurements.

The refinement on the CORELLI data shows the same amount of $\mathrm{Mn}-\mathrm{Sb}$ site mixing as determined from TOPAZ measurements (Table S5 in Supplemental Material [35]). The absence of structured diffuse scattering patterns suggests that the antisite defects do not induce significant local lattice distortions, and they are either randomly distributed or have a correlation length shorter than the probe limit $\approx 1 \mathrm{~nm}$. There is no magnetic Bragg scattering contribution for $\left(00 L\right.$ )-type peaks for both $\mathrm{T}_{N} 19 \mathrm{~K}$ and $\mathrm{T}_{C} 24 \mathrm{~K}$ (data not shown), suggesting that the ordered moment is along the $c$ axis. This is consistent with the anisotropic magnetic properties presented above. Figures 2(d) and 2(e) show the magnetic structures determined from the low-temperature diffraction datasets for $\mathrm{T}_{C} 24 \mathrm{~K}$ and $\mathrm{T}_{N} 19 \mathrm{~K}$, respectively. As shown in Fig. S3 in Supplemental Material, the magnetic order of $\mathrm{Mn}_{\mathrm{Sb}}^{\prime}$ ions has to be considered leading to the ferrimagnetic septuple layer for both magnetic structures. Both $\mathrm{Mn}_{\mathrm{Mn}}^{\times}$and $\mathrm{Mn}_{\mathrm{Sb}}^{\prime}$ ions carry significant ordered moments and they couple antiferromagnetically in each 

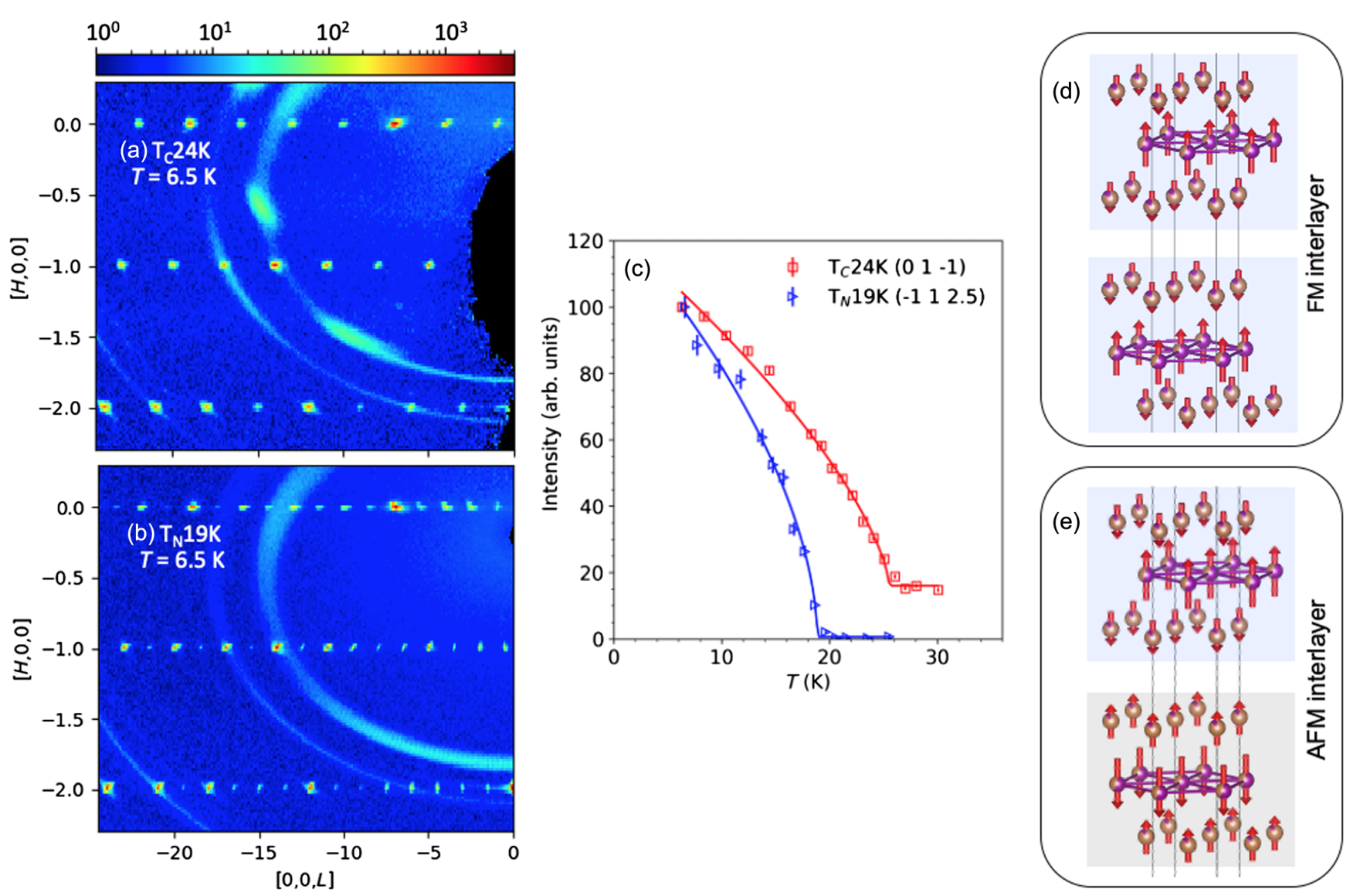

FIG. 2. Distinct interlayer coupling in different $\mathrm{MnSb}_{2} \mathrm{Te}_{4}$ single crystals resolved by single-crystal neutron diffraction. (a),(b) Neutron scattering pattern in the $[H 1 L]$ plane of the $\mathrm{T}_{C} 24 \mathrm{~K}$ and $\mathrm{T}_{N} 19 \mathrm{~K}$, respectively. The data were collected at $6.5 \mathrm{~K}$, where the sample $\mathrm{T}_{N} 19 \mathrm{~K}$ shows Bragg peaks at half- $L$ positions but $\mathrm{T}_{C} 24 \mathrm{~K}$ does not. (c) Temperature dependence of the normalized intensities of selected magnetic Bragg reflections for $\mathrm{T}_{N} 19 \mathrm{~K}$ and $\mathrm{T}_{C} 24 \mathrm{~K}$. The solid lines are guides to the eye. (d),(e) The schematic diagram of the magnetic structures of $\mathrm{T}_{C} 24 \mathrm{~K}$ and $\mathrm{T}_{N} 19 \mathrm{~K}$, respectively. Both have the same intraseptuple-layer ferrimagnetic order, but the FM interseptuplelayer coupling for $\mathrm{T}_{C} 24 \mathrm{~K}$ is in contrast to the $\mathrm{AFM}$ one for $\mathrm{T}_{N} 19 \mathrm{~K}$. The magnetic space groups for them are $R \overline{3} m^{\prime}$ (no. 166.101) and $R_{I}-3 c$ (no. 167.108), respectively. The sites connected by the purple triangular lattice represent the MnTe sheets while the other sites represent $\mathrm{Mn}$ mixed into the $\mathrm{Sb}_{2} \mathrm{Te}_{3}$ sheets.

septuple layer. At $6.5 \mathrm{~K}$, the ordered moment of $\approx 4.0 \mu_{B} / \mathrm{Mn}$ for $\mathrm{Mn}_{\mathrm{Mn}}^{\times}$is slightly larger than $\approx 3.5 \mu_{B} / \mathrm{Mn}$ for $\mathrm{Mn}_{\mathrm{Sb}}^{\prime}$. A distinct difference between the magnetic structures shown in Figs. 2(d) and 2(e) is the sign of the interseptuple-layer coupling. A FM interlayer coupling shown in Fig. 2(d) is in contrast to an AFM one in Fig. 2(e).

\section{Presence and random distribution of antisite defects from STEM and STM}

We further investigated the presence and distribution of $\mathrm{Mn}-\mathrm{Sb}$ antisites and looked for other potential lattice defects in $\mathrm{T}_{C} 24 \mathrm{~K}$ and $\mathrm{T}_{N} 19 \mathrm{~K}$ using STEM and STM. Figures 3(a) and 3(b) show the HAADF STEM images along the layer directions for $\mathrm{T}_{C} 24 \mathrm{~K}$ and $\mathrm{T}_{N} 19 \mathrm{~K}$, respectively, showing the Te-Sb-Te-Mn-Te-Sb-Te septuple layers stacking along the [001] direction. Since the HAADF STEM image intensity is roughly proportional to $Z^{2}(Z$ is the atomic number), the $\operatorname{Mn}(Z=25)$ atomic columns should show weaker intensity, while the Te $(Z=51)$ and Sb $(Z=52)$ atomic columns should display similar stronger intensities. However, the intensities of some Sb columns are weaker compared to the Te columns [in Figs. 3 (c) and 3(d)], indicating $\mathrm{Mn}_{\mathrm{Sb}}^{\prime}$ antisite defects. A careful inspection (Fig. S7 in Supplemental Material [35]) of the intensity variation of $\mathrm{Mn}$ and Te columns in (001) planes found a larger intensity variation among $\mathrm{Mn}$ atomic columns, indicating the partial substitution of $\mathrm{Mn}$ by heavier atoms. The STEM images for $\mathrm{T}_{C} 24 \mathrm{~K}$ and $\mathrm{T}_{N} 19 \mathrm{~K}$ look the same. No stacking faults or other lattice defects were identified.

The distribution of $\mathrm{Mn}$ at $\mathrm{Sb}$ site is further investigated by the STM measurement. The typical STM topographic images of $\mathrm{T}_{C} 24 \mathrm{~K}$ and $\mathrm{T}_{N} 19 \mathrm{~K}$ are shown in Figs. 3(e) and 3(f), respectively. In the fast Fourier transform in the insets, the sharp Bragg peaks indicate the atomic resolution of the images. The atomic resolution allows identification of $\mathrm{Mn}_{\mathrm{Sb}}^{\prime}$ and determination of its density on the surface. 

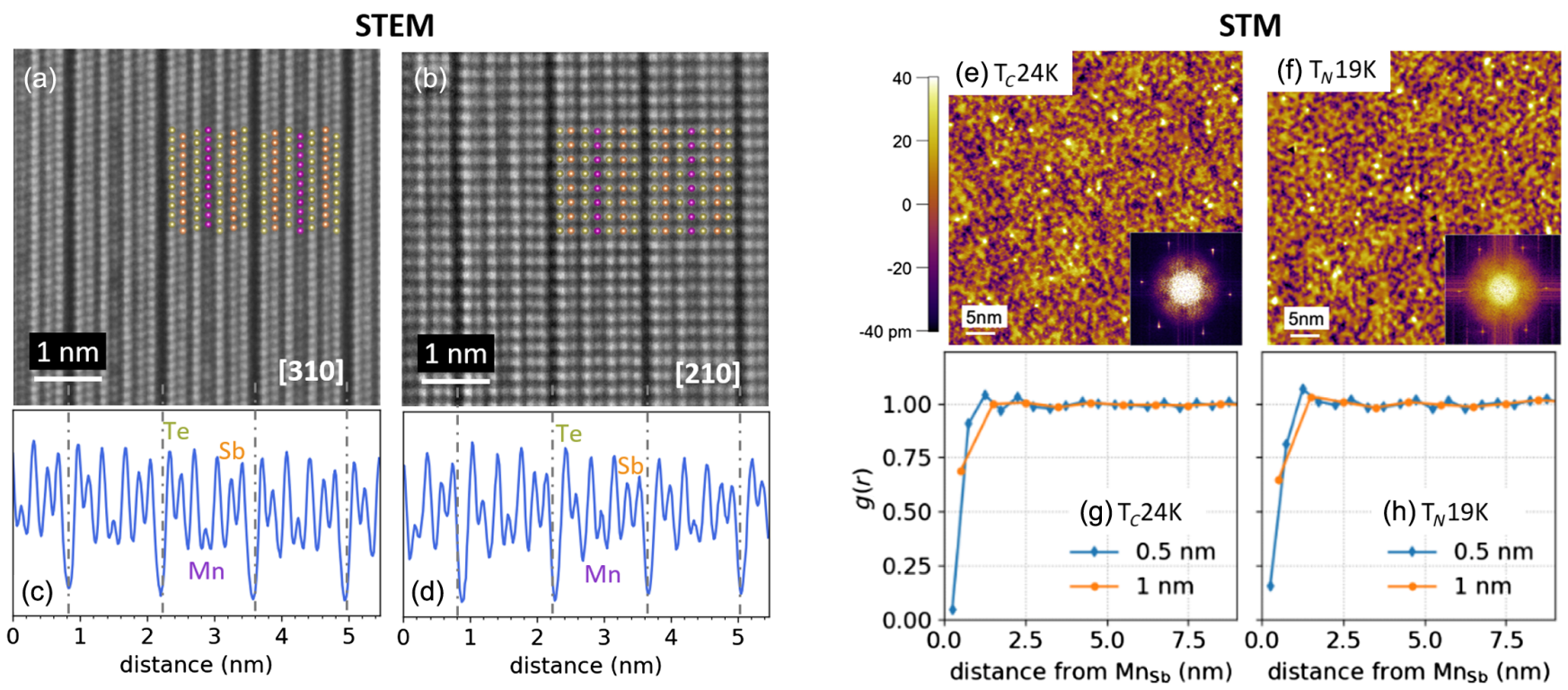

FIG. 3. Presence and random distribution of Mn-Sb antisite defects in both $\mathrm{MnSb}_{2} \mathrm{Te}_{4}$ crystals from STEM and STM. (a),(b) HAADF STEM images along [310] for $\mathrm{T}_{N} 19 \mathrm{~K}$ (a) and along [210] for $\mathrm{T}_{C} 24 \mathrm{~K}$ (b). No stacking fault was observed. (c),(d) Sum intensity profile perpendicular to the septuple layers. (e) STM topographic image of $\mathrm{T}_{C} 24 \mathrm{~K}$ (tunneling condition $-0.4 \mathrm{~V}, 100 \mathrm{pA}$ ). (f) STM topographic image of $\mathrm{T}_{N} 19 \mathrm{~K}$ (tunneling condition $-0.4 \mathrm{~V}, 60 \mathrm{pA}$ ). The insets in (e) and (f) are the fast Fourier transform of the topographic images. (g),(h) Radial pair distribution function of $\mathrm{Mn}_{\mathrm{Sb}}^{\prime}$ antisites. Radial pair distribution function $g(r)$ measures the normalized density of $\mathrm{Mn}_{\mathrm{Sb}}^{\prime}$ in the distance of $r$ away from a reference $\mathrm{Mn}_{\mathrm{Sb}}^{\prime}$. Two bin sizes, 0.5 and $1 \mathrm{~nm}$, are used to calculate $g(r)$.

Like $\mathrm{Mn}_{\mathrm{Bi}}^{\prime}$ in $\mathrm{MnBi}_{2} \mathrm{Te}_{4}[4,11], \mathrm{Mn}_{\mathrm{Sb}}^{\prime}$ in $\mathrm{MnSb}_{2} \mathrm{Te}_{4}$ exists as a dark triangle in STM topography due to the depression in the three nearest-neighbor Te atoms in the topmost plane right above the $\mathrm{Mn}_{\mathrm{Sb}}^{\prime}$. The density of $\mathrm{Mn}_{\mathrm{Sb}}^{\prime}$ is estimated to be $\approx 10.6 \%$ in $\mathrm{T}_{C} 24 \mathrm{~K}$, slightly larger than $\approx 8.4 \%$ in $\mathrm{T}_{N} 19 \mathrm{~K}$. The ratio is about 1.26 , consistent with our neutron scattering results. To explore the distribution of $\mathrm{Mn}_{\mathrm{Sb}}^{\prime}$, the radial distribution function analysis is performed after locating each $\mathrm{Mn}_{\mathrm{Sb}}^{\prime}$. The radial distribution function $g(r)$ describes how the density of defects varies as a function of distance, by calculating the normalized density of defects within a distance between $r-\frac{1}{2} d r$ and $r+\frac{1}{2} d r$ from a reference defect, where $d r$ is the bin size. Here two bin sizes of 0.5 and $1 \mathrm{~nm}$ are used. As shown in Figs. 3(g) and 3 (h), $g(r)$ is smaller than 1 at a short distance of $0.5 \mathrm{~nm}$, and fluctuates around 1 at larger distances, indicating some $\mathrm{Mn}_{\mathrm{Sb}}^{\prime}-\mathrm{Mn}_{\mathrm{Sb}}^{\prime}$ repulsion with a short correlation scale. In contrast, the clustering of defects would result in $g(r)$ much larger than 1 at short distances, which is absent in our analysis. A careful investigation of our STM images suggests $\approx 0.3 \%$ of $\mathrm{Sb}_{\mathrm{Te}}^{\prime}$ defects in both $\mathrm{T}_{C} 24 \mathrm{~K}$ and $\mathrm{T}_{N} 19 \mathrm{~K}$ crystals.

\section{Electronic structure calculations}

To understand the effects of site mixing on the magnetic order and electronic structure, we performed DFT calculations. We first constructed a $(2 \times 2 \times 2)$ rhombohedral supercell at stoichiometry with the $\mathrm{Mn}-\mathrm{Sb}$ site-mixing ratio of 50:50 in the central Mn layer and 25:75 in each of the
$\mathrm{Sb}$ layers. With the same alternating $\mathrm{Mn}$ and $\mathrm{Sb}$ rows in the central $\mathrm{Mn}$ layer in the $(2 \times 2 \times 2)$ supercell [see Figs. S8(b)-S8(f) in Supplemental Material [35]], all the possible five inequivalent configurations of one $\mathrm{Mn}$ mixed in each of the two Sb layers, or 25/75, have been considered. Figure 4(a) shows the most stable configuration in the $(2 \times 2 \times 2)$ supercell (labeled as AS3 in Table S7 and Fig. S8 in Supplemental Material) with the complex magnetic structure. Additionally, we also considered two configurations in the larger $(3 \times 3 \times 2)$ supercell with the Mn-Sb site-mixing ratio of 56:44 in the central layer and 22:78 in the Sb layer, where the central $\mathrm{Mn}$ layer has a mixture of $\mathrm{Mn}$ and $\mathrm{Sb}$ in each row, unlike the alternating rows in $(2 \times 2 \times 2)$. As listed in Table S7, after full relaxation in PEBsol $+U[48,50]$, a FM interlayer coupling is preferred for all the antisite configurations, in a distinct contrast to the ideal case without site mixing. Such a preference for all the configurations also holds when SOC is included during the full relaxation. As a reference, for the ideal $\mathrm{MnSb}_{2} \mathrm{Te}_{4}$ without site mixing, the A-type AFM structure is $3.48 \mathrm{meV} /$ f.u. more stable than the FM order for PBEsol $+U$ with relaxation. The $(2 \times 2 \times 2)$-AS3 complex magnetic configuration in Fig. 4(a) with a FM interlayer coupling is $0.31 \mathrm{meV} / \mathrm{f}$.u. more stable than that with an AFM interlayer coupling. Although in the above supercells the variation for composition and configuration is limited compared to the experimental compositions, these total energy differences confirm that the magnetic coupling between the septuple layers 
(a)
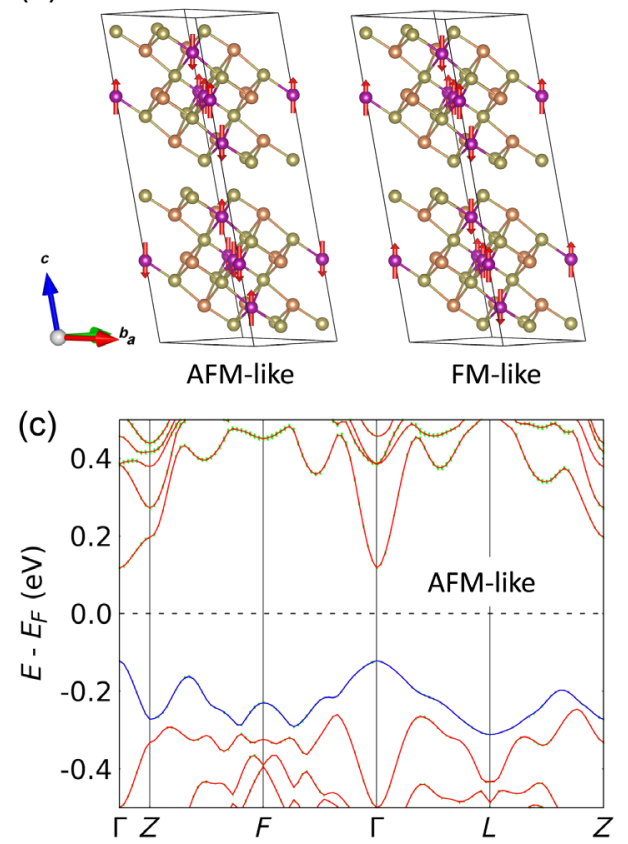

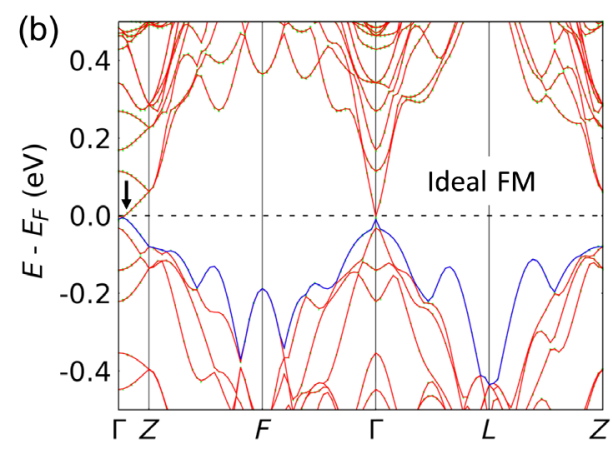

(d)

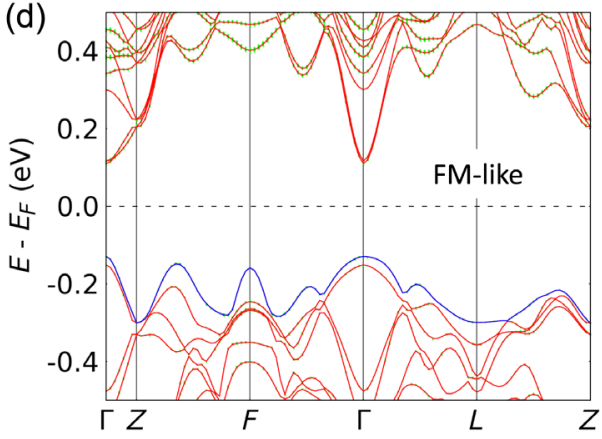

FIG. 4. Mn-Sb site mixing favors FM interlayer coupling but is detrimental to the band inversion from DFT. (a) $(2 \times 2 \times 2)$ supercells of rhombohedral $\mathrm{MnSb}_{2} \mathrm{Te}_{4}$ in the most stable configuration with the Mn-Sb site-mixing ratio of 50:50 in the central layer and 25:75 in the Sb layer, showing AFM or FM interlayer couplings while keeping the inversion symmetry. This configuration (labeled as AS3) and the other inequivalent ones are listed in Table S7 and shown in Fig. S8 of the Supplemental Material [35]. PBE $+U+\mathrm{SOC}$ band structures of (b) the ideal FM without site mixing, where the black arrow indicates the Weyl point in ideal FM along $\Gamma-Z$ due to band inversion. (c),(d) AFM-like and FM-like configurations with Mn-Sb site mixing. The top valence band is in blue and the green shadow indicates the projection on the $p$ orbital of $\mathrm{Sb}$ at $\mathrm{Mn}$ site.

changes the preference from AFM to FM with more Mn at $\mathrm{Sb}$ site, in qualitative agreement with experimental observation.

We further studied the effects of Mn-Sb site mixing on the electronic structure. First, our calculations show that the ideal $\mathrm{MnSb}_{2} \mathrm{Te}_{4}$ with an A-type AFM order has a small gap [34,51,52] without band inversion and is topologically trivial. In contrast, the ideal $\mathrm{MnSb}_{2} \mathrm{Te}_{4}$ with a FM order is topologically nontrivial supported by the Weyl point (black arrow) along $\Gamma-Z$ in Fig. 4(b) due to the band inversion between Sb-derived conduction and Te-derived valence band. Stabilizing FM in $\mathrm{MnSb}_{2} \mathrm{Te}_{4}$ is promising to realize magnetic Weyl points, similar to $\mathrm{EuCd}_{2} \mathrm{As}_{2}$ [53,54]. These results are consistent with previous reports [34,51,52]. We then introduced $\mathrm{Mn}-\mathrm{Sb}$ site mixing as discussed above, and the electronic band structures of the most stable configuration in the $(2 \times 2 \times 2)$ supercell with AFM and FM interlayer couplings are shown in Figs. 4(c) and 4(d), respectively. As shown in Fig. 4(c), each band is still doubly degenerated because the supercell retains inversion symmetry and the effective time-reversal symmetry [55]. The combined time-reversal operation and half translation along the $c$ axis is the same as in the ideal A-type AFM configuration. But the band gap is increased to $0.3 \mathrm{eV}$, the opposite direction for having a band inversion at $\Gamma$. With a FM interlayer coupling [see Fig. 4(d)], the band double degeneracy is lifted and the top valence band and bottom conduction band move toward each other reducing the band gap to $0.2 \mathrm{eV}$, albeit still no band inversion. Furthermore, from the total density of states (see Fig. S9 in Supplemental Material [35]) for all the site-mixing supercell configurations considered with both AFM- and FM-like coupling there is a bulk band gap of $0.2 \mathrm{eV}$ or beyond. Compared to the FM configuration of ideal $\mathrm{MnSb}_{2} \mathrm{Te}_{4}$, in contrast to the increased band gap at $\Gamma$ and $Z$ points, the gap at the $L$ point in Fig. 4(d) is reduced for $\mathrm{MnSb}_{2} \mathrm{Te}_{4}$ with significant site mixing. These band structure results indicate that although site mixing in $\mathrm{MnSb}_{2} \mathrm{Te}_{4}$ is beneficial to stabilize a $\mathrm{FM}$ interlayer coupling, it is detrimental to retain the band inversion needed for nontrivial topology. A high magnetic field is expected to ferromagnetically align Mn moments at both $\mathrm{Mn}$ and $\mathrm{Sb}$ sites. As shown in Fig. $\mathrm{S} 10$ in Supplemental Material, the calculation with all moments ferromagnetically aligned suggests still no band inversion although the band gap is slightly reduced compared to that in Fig. 4(d). To justify the calculations, Fig. S11 in Supplemental Material shows the scanning tunneling spectra (STS) data of $\mathrm{T}_{C} 24 \mathrm{~K}$ and $\mathrm{T}_{N} 19 \mathrm{~K}$ at $4.5 \mathrm{~K}$. Both samples have a bulk band gap of about $0.4 \mathrm{eV}$. The flat and diminished density of states within the band gap indicates the absence of in-gap states and trivial topology of the band structures. The calculated band gap in these supercell 
configurations agrees with the STS data, considering the underestimation of the band gap of the PBEsol exchangecorrelation functional.

\section{DISCUSSION}

\section{A. Effects of site mixing on the magnetic and electronic properties of $\mathrm{MnSb}_{2} \mathrm{Te}_{4}$}

Our results show that magnetic defects can have a dramatic effect on the magnetic ordering in $\mathrm{MnSb}_{2} \mathrm{Te}_{4}$, and $\mathrm{Mn}-\mathrm{Sb}$ site mixing favors a FM interlayer coupling. The anisotropic magnetic properties shown in Fig. 1 demonstrate that the interseptuple-layer coupling can be FM or AFM, depending on the crystal growth parameters. Our neutron diffraction and microscopy measurements found a significant amount of $\mathrm{Mn}-\mathrm{Sb}$ site mixing with nearly random distribution in all crystals investigated in this work. We noticed that similar Mn-Sb site mixing is also observed in a polycrystalline sample with a FM interlayer coupling by neutron powder diffraction [34] and in a single-crystal sample by single-crystal x-ray diffraction [51]. The observation of $\mathrm{Mn}-\mathrm{Sb}$ site mixing in $\mathrm{MnSb}_{2} \mathrm{Te}_{4}$ samples synthesized differently by different groups suggests that the presence of $\mathrm{Mn}-\mathrm{Sb}$ mixing is a general phenomenon in $\mathrm{MnSb}_{2} \mathrm{Te}_{4}$, possibly due to the small formation energy of antisite defects [15]. By investigating the anisotropic magnetic properties, concentration, and distribution of $\mathrm{Mn}-\mathrm{Sb}$ site mixing, this work develops a direct correlation between the magnetic ground states and magnetic defects. Other intrinsic lattice defects, such as $\mathrm{Sb}_{\mathrm{Te}}^{\prime}$ and $\mathrm{Te}_{\mathrm{Sb}}^{\cdot}$, might also exist with a smaller concentration and mainly affect the transport properties. Therefore, the formation of the complex magnetic structure and the sign change of interseptuple-layer coupling result from the occupation of $\mathrm{Mn}$ at $\mathrm{Sb}$ sites. We noticed that with increasing growth temperature, both $\mathrm{Mn}_{\mathrm{Mn}}^{\times}$and $\mathrm{Mn}_{\mathrm{Sb}}^{\prime}$ increase in concentration, and hence the total Mn content. The difference between $\mathrm{T}_{C} 24 \mathrm{~K}$ and $\mathrm{T}_{N} 19 \mathrm{~K}$ suggests a threshold concentration of $13 \% \mathrm{Mn}_{\mathrm{Sb}}^{\prime}$, above which the interseptuple-layer interaction becomes FM. With further increasing the total $\mathrm{Mn}$ content, the magnetic ordering temperature increases to about $50 \mathrm{~K}$, as illustrated in Fig. S4 in Supplemental Material [35]. Our results demonstrate that both the sign of interlayer coupling and the magnetic ordering temperature are tunable by a careful control of the concentration and distribution of magnetic ions in the lattice.

The modification of the magnetic ground state results from the additional competing interactions introduced by $\mathrm{Mn}_{\mathrm{Sb}}^{\prime}$. The energy difference between FM and AFM states is small in both ideal $\mathrm{MnBi}_{2} \mathrm{Te}_{4}$ and $\mathrm{MnSb}_{2} \mathrm{Te}_{4}$. Therefore, the magnetic ground state can be sensitive to external perturbations. The competing interactions in $\mathrm{MnBi}_{2} \mathrm{Te}_{4}$ were studied previously using inelastic neutron scattering $[16,17]$. Similar competing interactions are expected in ideal $\mathrm{MnSb}_{2} \mathrm{Te}_{4}$. The presence of $\mathrm{Mn}_{\mathrm{Sb}}^{\prime}$ introduces additional competing magnetic interactions. Considering a FM order is always observed in $T M$-doped ( $T M=\mathrm{Mn}, \mathrm{Cr}$, and V) $\mathrm{Bi}_{2} \mathrm{Te}_{3}$ and $\mathrm{Sb}_{2} \mathrm{Te}_{3}$, the interaction between neighboring $\mathrm{Mn}_{\mathrm{Sb}}^{\prime}$ and $\mathrm{Mn}_{\mathrm{Sb}}^{\prime}$ ions right across the van der Waals gap is expected to be FM. This FM interaction increases in magnitude with increasing amount of $\mathrm{Mn}_{\mathrm{Sb}}^{\prime}$ and competes with the AFM interaction between $M_{M n}^{\times}$and $M_{M n}^{\times}$in neighboring septuple layers and eventually leads to the sign change of interlayer magnetic coupling. Other interactions between further neighboring $\mathrm{Mn}$ ions will also contribute to the overall interlayer magnetic interactions but are possibly less important. $\mathrm{Mn}_{\mathrm{Sb}}^{\prime}$ magnetic defects also introduce additional exchange interactions in each septuple layer. Both $90^{\circ}$ and $180^{\circ} \mathrm{Mn}-\mathrm{Te}-\mathrm{Mn}$ superexchange interactions are active and contribute to the overall interaction between $\mathrm{Mn}_{\mathrm{Sb}}^{\prime}$ and $\mathrm{Mn}_{\mathrm{Mn}}^{\times}$. While the partial contribution of different exchange paths to the total exchange is to be investigated, the experimentally observed ferrimagnetic arrangement in each septuple layer suggests an AFM interaction between $\mathrm{Mn}_{\mathrm{Sb}}^{\prime}$ and $\mathrm{Mn}_{\mathrm{Mn}}^{\times}$. As described later, this AFM interaction is rather strong.

$\mathrm{Mn}$-Sb site mixing favors a FM interlayer coupling but is detrimental to the band inversion. Our DFT calculations considered different antisite configurations using the $(2 \times 2 \times 2)$ supercell and found all antisite configurations prefer a FM interlayer coupling. This is further confirmed by using a larger supercell $(3 \times 3 \times 2)$. This FM interlayer coupling is preferred for the realization of a Weyl state. Unfortunately, our band structure calculations suggest a sizable band gap of $>0.2 \mathrm{eV}$ regardless of the sign of interlayer magnetic interaction or the antisite configuration. STS measurement further confirms the sizable gap in our crystals with significant amount of $\mathrm{Mn}-\mathrm{Sb}$ site mixing. The site-mixing-induced gap opening is also reproduced in a most recent study by Wimmer et al. [56]. In their DFT calculations, they considered the effect of $20 \% \mathrm{Mn}-\mathrm{Sb}$ site mixing on the band structure and found a gap of about $0.2 \mathrm{eV}$, which makes $\mathrm{MnSb}_{2} \mathrm{Te}_{4}$ to be topological trivial. In addition, Wimmer et al. also considered the effect of $5 \% \mathrm{Mn}-\mathrm{Sb}$ antisite mixing on the band structure of $\mathrm{FM} \mathrm{MnSb} \mathrm{Te}_{4}$ to find that the band inversion and Weyl point are preserved. This interesting result indicates that it is possible to tune the magnetism by introducing $\mathrm{Mn}-\mathrm{Sb}$ (or $\mathrm{Mn}-\mathrm{Bi}$ ) site mixing in $\mathrm{MnSb}_{2} \mathrm{Te}_{4}$ (or $\mathrm{MnBi}_{2} \mathrm{Te}_{4}$ ) without losing the band inversion, as suggested below.

\section{B. Indications for $\operatorname{MnTe}\left(\mathrm{Bi}_{2} \mathbf{T e}_{3}\right)_{n}$}

Since about $3 \% \mathrm{Mn}_{\mathrm{Bi}}^{\prime}$ ions were observed in $\mathrm{MnBi}_{2} \mathrm{Te}_{4}$ single crystals by different groups, one naturally asks (1) whether those $3 \% \mathrm{Mn}_{\mathrm{Bi}}^{\prime}$ ions order magnetically forming ferrimagnetic septuple layers as observed in $\mathrm{MnSb}_{2} \mathrm{Te}_{4}$, (2) whether it is possible to tune the magnetic ground state, and thus the topological properties, by controlling the concentration and distribution of magnetic 
defects in $\mathrm{MnBi}_{2} \mathrm{Te}_{4}$, and (3) whether the site mixing plays any role in other $n>1$ compounds in the $\mathrm{MnTe}\left(\mathrm{Bi}_{2} \mathrm{Te}_{3}\right)_{n}$ family with more complex stacking of the septuple and quintuple layers. As discussed below, $\mathrm{Mn}_{\mathrm{Bi}}^{\prime}$ ions likely order magnetically as observed in $\mathrm{MnSb}_{2} \mathrm{Te}_{4}$, and the magnetism of $\mathrm{Mn}_{\mathrm{Bi}}^{\prime}$, in either the septuple or the quintuple layers, should be considered in all $\operatorname{MnTe}\left(\mathrm{Bi}_{2} \mathrm{Te}_{3}\right)_{n}$ compounds.

We noticed that the growth-dependent magnetic behavior as shown in Fig. 1 is absent in $\mathrm{MnBi}_{2} \mathrm{Te}_{4}$. All previous work reported an AFM order with $T_{N}=25 \mathrm{~K}$ for $\mathrm{MnBi}_{2} \mathrm{Te}_{4}$ crystals. This consistency might result from the narrow crystallization temperature window and/or the limited concentration of magnetic defects due to the large difference of ionic size and electronegativity between Mn and $\mathrm{Bi}$. Considering possible AFM alignment of $\mathrm{Mn}_{\mathrm{Bi}}^{\prime}$ and $\mathrm{Mn}_{\mathrm{Mn}}^{\times}$, the field dependence of magnetization can provide more valuable information.

As proposed by Murakami et al. [34], the ferrimagnetic arrangement of $\mathrm{Mn}$ moments in each septuple layer of $\mathrm{MnSb}_{2} \mathrm{Te}_{4}$ provides a straightforward explanation for the large difference between the ordered and saturation moments. With the ordered moment at $6.5 \mathrm{~K}$ and the occupancy both determined from neutron single-crystal diffraction, the average moment within each septuple layer is $1.52(4)$ and $1.65(8) \mu_{B} / \mathrm{Mn}$ at $6.5 \mathrm{~K}$ for $\mathrm{T}_{N} 19 \mathrm{~K}$ and $\mathrm{T}_{C} 24 \mathrm{~K}$, respectively (Table S6 in Supplemental Material [35]). These values are smaller than the saturation moment determined at $2 \mathrm{~K}$ from magnetic measurements but in reasonable agreement. The magnetization at $2 \mathrm{~K}$ does not saturate even in an applied magnetic field of $120 \mathrm{kOe}$, indicating a strong magnetic coupling between $\mathrm{Mn}_{\mathrm{Mn}}^{\times}$and $\mathrm{Mn}_{\mathrm{Sb}}^{\prime}$. A field-induced transition to a state with all $\mathrm{Mn}$ moments polarized is expected at a much higher magnetic field, and this deserves further study.

One important indication of the above analyses is possible magnetic order of $\mathrm{Mn}_{\mathrm{Bi}}^{\prime}$ ions in $\mathrm{MnBi}_{2} \mathrm{Te}_{4}$. The saturation moment of $\mathrm{MnBi}_{2} \mathrm{Te}_{4}$ is $\approx 3.8 \mu_{B} / \mathrm{Mn}$ estimated from magnetic measurements at $2 \mathrm{~K}[4,16,33]$, which is smaller than the ordered moment of $4.04(13) \mu_{B} / \mathrm{Mn}$ at $10 \mathrm{~K}$ [4] or 4.7(1) $\mu_{B} / \mathrm{Mn}$ at $4.5 \mathrm{~K}$ [57] determined from neutron diffraction. The amount of $\mathrm{Mn}_{\mathrm{Bi}}^{\prime}$ is $\approx 3 \%$ in our crystals [4]. With the assumption that $\mathrm{Mn}_{\mathrm{Mn}}^{\times}$and $\mathrm{Mn}_{\mathrm{Bi}}^{\prime}$ ions are antiferromagnetically aligned with the same ordered moment of $4.7 \mu_{B}$, the net moment of each septuple layer is about $4.1 \mu_{B}$, in reasonable agreement with the experimentally measured saturation moment at $2 \mathrm{~K}$. It should be noted that $2 \% \mathrm{Mn}$ on $\mathrm{Bi}$ sites is enough to induce a longrange $\mathrm{FM}$ order in $\mathrm{Bi}_{2} \mathrm{Te}_{3}$. From these points of view, the magnetic structure of $\mathrm{MnBi}_{2} \mathrm{Te}_{4}$ and its field dependence should be carefully revisited. Considering the similarity between $\mathrm{MnBi}_{2} \mathrm{Te}_{4}$ and $\mathrm{MnSb}_{2} \mathrm{Te}_{4}$, a similar complex ferrimagnetic structure is expected for $\mathrm{MnBi}_{2} \mathrm{Te}_{4}$ if $\mathrm{Mn}_{\mathrm{Bi}}^{\prime}$ ions form a long-range magnetic order below $T_{N}=25 \mathrm{~K}$.
The effects of magnetic defects from site mixing should be considered in the entire family of $\mathrm{MnTe}\left(\mathrm{Bi}_{2} \mathrm{Te}_{3}\right)_{n}$, its $\mathrm{Sb}$ analogs, and their solid solution. The doping dependence of magnetization in $\mathrm{MnBi}_{2-x} \mathrm{Sb}_{x} \mathrm{Te}_{4}$ was carefully studied previously [33]. The gradually suppressed saturation moment with increasing $\mathrm{Sb}$ doping signals the increasing amount of $\mathrm{Mn}$ at $\mathrm{Bi}-\mathrm{Sb}$ site. The evolution with doping of the magnetic and topological properties should be revisited in this system by considering the doping-dependent site mixing and the magnetic ordering of antisite Mn ions. The magnetic order of antisite $\mathrm{Mn}$ ions will impact the surface magnetism, and of particular interest are whether and how this magnetic order couples to the topological surface states which deserve further investigation. Similar studies are also relevant for $\operatorname{MnTe}\left(\mathrm{Bi}_{2} \mathrm{Te}_{3}\right)_{n}$ compounds with $n>1$ considering the presence of $\mathrm{Mn}_{\mathrm{Bi}}^{\prime}$ in both the quintuple and septuple layers. STM studies observed $2 \%-3 \% \mathrm{Mn}_{\mathrm{Bi}}^{\prime}$ ions in both the quintuple and septuple layers in $\mathrm{MnBi}_{4} \mathrm{Te}_{7}$ crystals $[30,32]$.

Understanding the effects of magnetic defects suggests new approaches toward fine tuning of the magnetic and topological properties of $\mathrm{MnBi}_{2} \mathrm{Te}_{4}$ and related compounds. Besides the natural antisite defects, one can purposely introduce magnetic ions, such as $\mathrm{V}$ or $\mathrm{Cr}$, at $\mathrm{Bi}$ site to tune the magnetism and even change the sign of interseptuple-layer magnetic interaction in $\mathrm{MnBi}_{2} \mathrm{Te}_{4}$. Realization of such a complex magnetic structure without losing the band inversion might be a valid approach toward QAHE in $\mathrm{MnBi}_{2} \mathrm{Te}_{4}$. On the other hand, reducing the amount of site mixing might lead to quantized transport properties at even higher temperatures than reported in $\mathrm{MnBi}_{2} \mathrm{Te}_{4}$ flakes [58-61]. Considering the narrow crystallization temperature window for the flux growth of $\mathrm{MnBi}_{2} \mathrm{Te}_{4}$ out of $\mathrm{Bi}-\mathrm{Te}$ flux, vapor transport growth of bulk crystals or MBE growth of thin films might have a better control of the concentration of magnetic ions on Bi sites.

\section{SUMMARY}

In summary, we have demonstrated the importance of understanding and controlling the defect concentration and distribution in any candidate magnetic topological insulators. In particular, we have shown that $\mathrm{Mn}-\mathrm{Sb}$ site mixing can have a dramatic effect on the magnetic ground state and electronic structure of $\mathrm{MnSb}_{2} \mathrm{Te}_{4}$. These antisite defects favor a FM interlayer coupling. While an ideal FM $\mathrm{MnSb}_{2} \mathrm{Te}_{4}$ crystal hosts Weyl points, site mixing is detrimental to the desired nontrivial band topology. Our work highlights the importance of further investigating lattice defects in the isostructural $\mathrm{MnBi}_{2} \mathrm{Te}_{4}$ and related compounds, where Mn-Bi site mixing has been observed although by a much reduced amount. How the magnetic defects $\mathrm{Mn}_{\mathrm{Bi}}^{\prime}$ in $\operatorname{MnTe}\left(\mathrm{Bi}_{2} \mathrm{Te}_{3}\right)_{n}$ affect the surface magnetism and interact with the topological surface states deserves further study. 


\section{ACKNOWLEDGMENTS}

The authors thank Huibo Cao, Maohua Du, Robert McQueeney, Satoshi Okamoto, and Xiaodong $\mathrm{Xu}$ for helpful discussions and Michael Lance for WDS measurements. Research at ORNL (M. A. M., B. C. S., and J. Y.) was supported by the U.S. Department of Energy, Office of Science, Basic Energy Sciences, Division of Materials Science and Engineering. L.-L. W. was supported by the Center for the Advancement of Topological Semimetals, an Energy Frontier Research Center funded by U.S. Department of Energy (DOE), Office of Science, Basic Energy Sciences. Ames Laboratory is operated for the U.S. Department of Energy by Iowa State University under Contract No. DE-AC02-07CH11358. Q. Z. acknowledges support from the Gordon and Betty Moore Foundations EPiQS Initiative, Grant No. GBMF9069. The STM work at Rutgers was supported by ARO Grant No. W911NF2010108. M.C. is supported by an Early Career project supported by DOE Office of Science. A portion of this research used resources at the High Flux Isotope Reactor, Spallation Neutron Source and the Center for Nanophase Materials Sciences, DOE Office of Science User Facilities operated by the Oak Ridge National Laboratory.

[1] C.-Z. Chang, J. Zhang, X. Feng, J. Shen, Z. Zhang, M. Guo, K. Li, Y. Ou, P. Wei, L.-L. Wang et al., Experimental Observation of the Quantum Anomalous Hall Effect in a Magnetic Topological Insulator, Science 340, 167 (2013).

[2] D. S. Lee, T.-H. Kim, C.-H. Park, C.-Y. Chung, Y. S. Lim, W.-S. Seo, and H.-H. Park, Crystal Structure, Properties and Nanostructuring of a New Layered Chalcogenide Semiconductor, $\mathrm{Bi}_{2} \mathrm{MnTe}_{4}$, Cryst. Eng. Commun. 15, 5532 (2013).

[3] M. M. Otrokov, I. I. Klimovskikh, H. Bentmann, D. Estyunin, A. Zeugner, Z.S. Aliev, S. Gaß, A. Wolter, A. Koroleva, A. M. Shikin et al., Prediction and Observation of an Antiferromagnetic Topological Insulator, Nature (London) 576, 416 (2019).

[4] J.-Q. Yan, Q. Zhang, T. Heitmann, Z. Huang, K. Y. Chen, J.-G. Cheng, W. Wu, D. Vaknin, B. C. Sales, and R. J. McQueeney, Crystal Growth and Magnetic Structure of $\mathrm{MnBi}_{2} \mathrm{Te}_{4}$, Phys. Rev. Mater. 3, 064202 (2019).

[5] D. Scanlon, P. King, R. P. Singh, A. De La Torre, S. M. Walker, G. Balakrishnan, F. Baumberger, and C. Catlow, Controlling Bulk Conductivity in Topological Insulators: Key Role of Anti-Site Defects, Adv. Mater. 24, 2154 (2012).

[6] R. J. Cava, H. Ji, M. K. Fuccillo, Q. D. Gibson, and Y. S. Hor, Crystal Structure and Chemistry of Topological Insulators, J. Mater. Chem. C 1, 3176 (2013).

[7] Y. Ando, Topological Insulator Materials, J. Phys. Soc. Jpn. 82, 102001 (2013).
[8] A. Hashibon and C. Elsässer, First-Principles Density Functional Theory Study of Native Point Defects in $\mathrm{Bi}_{2} \mathrm{Te}_{3}$, Phys. Rev. B 84, 144117 (2011).

[9] B. Zhou, Z. Liu, J. Analytis, K. Igarashi, S. Mo, D. Lu, R. Moore, I. Fisher, T. Sasagawa, Z. Shen et al., Controlling the Carriers of Topological Insulators by Bulk and Surface Doping, Semicond. Sci. Technol. 27, 124002 (2012).

[10] P.-Y. Chuang, S.-H. Su, C.-W. Chong, Y.-F. Chen, Y.-H. Chou, W.-C. Chen, C.-M. Cheng, K.-D. Tsuei, C.-H. Wang, Y.-W. Yang et al., Anti-Site Defect Effect on the Electronic Structure of a $\mathrm{Bi}_{2} \mathrm{Te}_{3}$ Topological Insulator, RSC Adv. 8, 423 (2018).

[11] Y. Yuan, X. Wang, H. Li, J. Li, Y. Ji, Z. Hao, Y. Wu, K. He, Y. Wang, Y. Xu et al., Electronic States and Magnetic Response of $\mathrm{MnBi}_{2} \mathrm{Te}_{4}$ by Scanning Tunneling Microscopy and Spectroscopy, Nano Lett. 20, 3271 (2020).

[12] K. Zhu, Y. Bai, X. Hong, Z. Geng, Y. Jiang, R. Liu, Y. Li, M. Shi, L. Wang, W. Li et al., Investigating and Manipulating the Molecular Beam Epitaxy Growth Kinetics of Intrinsic Magnetic Topological Insulator $\mathrm{MnBi}_{2} \mathrm{Te}_{4}$ with In-Situ Angle-Resolved Photoemission Spectroscopy, J. Phys. Condens. Matter 32, 475002 (2020).

[13] A. Zeugner, F. Nietschke, A. U. Wolter, S. Gaß, R. C. Vidal, T. R. Peixoto, D. Pohl, C. Damm, A. Lubk, R. Hentrich et al., Chemical Aspects of the Candidate Antiferromagnetic Topological Insulator $\mathrm{MnBi}_{2} \mathrm{Te}_{4}$, Chem. Mater. 31, 2795 (2019).

[14] H. Li, S. Liu, C. Liu, J. Zhang, Y. Xu, R. Yu, Y. Wu, Y. Zhang, and S. Fan, Antiferromagnetic Topological Insulator $\mathrm{MnBi}_{2} \mathrm{Te}_{4}$ : Synthesis and Magnetic Properties, Phys. Chem. Chem. Phys. 22, 556 (2020).

[15] M.-H. Du, J. Yan, V. R. Cooper, and M. Eisenbach, Tuning Fermi Levels in Intrinsic Antiferromagnetic Topological Insulators $\mathrm{MnBi}_{2} \mathrm{Te}_{4}$ and $\mathrm{MnBi}_{4} \mathrm{Te}_{7}$ by Defect Engineering and Chemical Doping, Adv. Funct. Mater. 31, 2006516 (2021).

[16] B. Li, J.-Q. Yan, D. M. Pajerowski, E. Gordon, A.-M. Nedić, Y. Sizyuk, L. Ke, P. P. Orth, D. Vaknin, and R. J. McQueeney, Competing Magnetic Interactions in the Antiferromagnetic Topological Insulator $\mathrm{MnBi}_{2} \mathrm{Te}_{4}$, Phys. Rev. Lett. 124, 167204 (2020).

[17] B. Li, D. Pajerowski, S. Riberolles, L. Ke, J. Yan, and R. McQueeney, Two-Dimensional Ferromagnetism with LongRange Interactions in the Layered Magnetic Topological Insulator $\mathrm{MnBi}_{2} \mathrm{Te}_{4}$, arXiv:2007.08468.

[18] S. H. Lee, Y. Zhu, Y. Wang, L. Miao, T. Pillsbury, H. Yi, S. Kempinger, J. Hu, C. A. Heikes, P. Quarterman et al., Spin Scattering and Noncollinear Spin Structure-Induced Intrinsic Anomalous Hall Effect in Antiferromagnetic Topological Insulator $\mathrm{MnBi}_{2} \mathrm{Te}_{4}$, Phys. Rev. Research 1, 012011(R) (2019).

[19] P. Swatek, Y. Wu, L.-L. Wang, K. Lee, B. Schrunk, J. Yan, and A. Kaminski, Gapless Dirac Surface States in the Antiferromagnetic Topological Insulator $\mathrm{MnBi}_{2} \mathrm{Te}_{4}$, Phys. Rev. B 101, 161109(R) (2020).

[20] R. C. Vidal, H. Bentmann, T. R. F. Peixoto, A. Zeugner, S. Moser, C.-H. Min, S. Schatz, K. Kißner, M. Ünzelmann, C. I. Fornari et al., Surface States and Rashba-Type Spin Polarization in Antiferromagnetic $\mathrm{MnBi}_{2} \mathrm{Te}_{4}(0001)$, Phys. Rev. B 100, 121104(R) (2019). 
[21] Y. J. Chen, L. X. Xu, J. H. Li, Y. W. Li, H. Y. Wang, C. F. Zhang, H. Li, Y. Wu, A. J. Liang, C. Chen et al., Topological Electronic Structure and Its Temperature Evolution in Antiferromagnetic Topological Insulator $\mathrm{MnBi}_{2} \mathrm{Te}_{4}$, Phys. Rev. X 9, 041040 (2019).

[22] H. Li, S.-Y. Gao, S.-F. Duan, Y.-F. Xu, K.-J. Zhu, S.-J. Tian, J.-C. Gao, W.-H. Fan, Z.-C. Rao, J.-R. Huang et al., Dirac Surface States in Intrinsic Magnetic Topological Insulators $\mathrm{EuSn}_{2} \mathrm{As}_{2}$ and $\mathrm{MnBi}_{2 n} \mathrm{Te}_{3 n+1}$, Phys. Rev. X 9, 041039 (2019).

[23] Y.-J. Hao, P. Liu, Y. Feng, X.-M. Ma, E. F. Schwier, M. Arita, S. Kumar, C. Hu, R. Lu, M. Zeng, Y. Wang et al., Gapless Surface Dirac Cone in Antiferromagnetic Topological Insulator $\mathrm{MnBi}_{2} \mathrm{Te}_{4}$, Phys. Rev. X 9, 041038 (2019).

[24] D. Souchay, M. Nentwig, D. Günther, S. Keilholz, J. de Boor, A. Zeugner, A. Isaeva, M. Ruck, A. U. Wolter, B. Büchner et al., Layered Manganese Bismuth Tellurides with $\mathrm{GeBi}_{4} \mathrm{Te}_{7}$ - and $\mathrm{GeBi}_{6} \mathrm{Te}_{10}$-Type Structures: Towards Multifunctional Materials, J. Mater. Chem. C 7, 9939 (2019).

[25] J. Wu, F. Liu, M. Sasase, K. Ienaga, Y. Obata, R. Yukawa, K. Horiba, H. Kumigashira, S. Okuma, T. Inoshita et al., Natural van der waals Heterostructural Single Crystals with Both Magnetic and Topological Properties, Sci. Adv. 5, eaax9989 (2019).

[26] J.-Q. Yan, Y. H. Liu, D. S. Parker, Y. Wu, A. A. Aczel, M. Matsuda, M. A. McGuire, and B. C. Sales, A-Type Antiferromagnetic Order in $\mathrm{MnBi}_{4} \mathrm{Te}_{7}$ and $\mathrm{MnBi}_{6} \mathrm{Te}_{10}$ Single Crystals, Phys. Rev. Mater. 4, 054202 (2020).

[27] R. C. Vidal, A. Zeugner, J. I. Facio, R. Ray, M. H. Haghighi, A. U. B. Wolter, L. T. C. Bohorquez, F. Caglieris, S. Moser, T. Figgemeier et al., Topological Electronic Structure and Intrinsic Magnetization in $\mathrm{MnBi}_{4} \mathrm{Te}_{7}: A \mathrm{Bi}_{2} \mathrm{Te}_{3}$ Derivative with a Periodic Mn Sublattice, Phys. Rev. X 9, 041065 (2019).

[28] C. Hu, L. Ding, K. N. Gordon, B. Ghosh, H. Tien, H. Li, A. G. Linn, S. Lien, C.-Y. Huang, S. Mackey et al., Realization of an Intrinsic Ferromagnetic Topological State in $\mathrm{MnBi}_{8} \mathrm{Te}_{13}$, Sci. Adv. 6, eaba4275 (2020).

[29] S. Tian, S. Gao, S. Nie, Y. Qian, C. Gong, Y. Fu, H. Li, W. Fan, P. Zhang, T. Kondo et al., Magnetic Topological Insulator in $\mathrm{MnBi}_{6} \mathrm{Te}_{10}$ with a Zero-Field Ferromagnetic State and Gapped Dirac Surface States, Phys. Rev. B 102, 035144 (2020).

[30] Z. Liang, A. Luo, M. Shi, Q. Zhang, S. Nie, J. J. Ying, J.-F. He, T. Wu, Z. Wang, G. Xu, and X.-H. Chen, Mapping Dirac Fermions in the Intrinsic Antiferromagnetic Topological Insulators $\left(\mathrm{MnBi}_{2} \mathrm{Te}_{4}\right)\left(\mathrm{Bi}_{2} \mathrm{Te}_{3}\right)_{n}(n=0,1)$, Phys. Rev. B 102, 161115(R) (2020).

[31] J. Wu, F. Liu, C. Liu, Y. Wang, C. Li, Y. Lu, S. Matsuishi, and $\mathrm{H}$. Hosono, Toward 2D Magnets in the $\left(\mathrm{MnBi}_{2} \mathrm{Te}_{4}\right)\left(\mathrm{Bi}_{2} \mathrm{Te}_{3}\right)_{n}$ Bulk Crystal, Adv. Mater. 32, 2001815 (2020).

[32] X. Wu, J. Li, X.-M. Ma, Y. Zhang, Y. Liu, C.-S. Zhou, J. Shao, Q. Wang, Y.-J. Hao, Y. Feng et al., Distinct Topological Surface States on the Two Terminations of $\mathrm{MnBi}_{4} \mathrm{Te}_{7}$, Phys. Rev. X 10, 031013 (2020).

[33] J.-Q. Yan, S. Okamoto, M. A. McGuire, A. F. May, R. J. McQueeney, and B.C. Sales, Evolution of Structural,
Magnetic, and Transport Properties in $\mathrm{MnBi}_{2-x} \mathrm{Sb}_{x} \mathrm{Te}_{4}$, Phys. Rev. B 100, 104409 (2019).

[34] T. Murakami, Y. Nambu, T. Koretsune, G. Xiangyu, T. Yamamoto, C. M. Brown, and H. Kageyama, Realization of Interlayer Ferromagnetic Interaction in $\mathrm{MnSb}_{2} \mathrm{Te}_{4}$ toward the Magnetic Weyl Semimetal State, Phys. Rev. B 100, 195103 (2019).

[35] See Supplemental Material at http://link.aps.org/ supplemental/10.1103/PhysRevX.11.021033 for (1) refinement of nuclear and magnetic structures, (2) tunable magnetic ordering temperatures, (3) transport properties, (4) DFT calculations of different supercell configurations with site mixing, and (5) STS measurement of bulk gap.

[36] T. M. Michels-Clark, A. T. Savici, V. E. Lynch, X. Wang, and C. M. Hoffmann, Expanding Lorentz and Spectrum Corrections to Large Volumes of Reciprocal Space for Single-Crystal Time-of-Flight Neutron Diffraction, J. Appl. Crystallogr. 49, 497 (2016).

[37] A. J. Schultz, K. Srinivasan, R. G. Teller, J. M. Williams, and C. Lukehart, Single-Crystal, Time-of-Flight, NeutronDiffraction Structure of Hydrogen cis-Diacetyltetracarbonylrhenate, $\left[\right.$ cis- $\left.(\mathrm{OC})_{4} \operatorname{Re}\left(\mathrm{Ch}_{3} \mathrm{Co}\right)_{2}\right] \mathrm{H}$ : A Metallaacetylacetone Molecule, J. Am. Chem. Soc. 106, 999 (1984).

[38] G. M. Sheldrick, A Short History of SHELX, Acta Crystallogr. Sect. A 64, 112 (2008).

[39] F. Ye, Y. Liu, R. Whitfield, R. Osborn, and S. Rosenkranz, Implementation of Cross Correlation for Energy Discrimination on the Time-of-Flight Spectrometer Corelli, J. Appl. Crystallogr. 51, 315 (2018).

[40] A. Wills, A New Protocol for the Determination of Magnetic Structures Using Simulated Annealing and Representational Analysis (SARAh), Physica (Amsterdam) 276B, 680 (2000).

[41] J. Perez-Mato, S. Gallego, E. Tasci, L. Elcoro, G. de la Flor, and M. Aroyo, Symmetry-Based Computational Tools for Magnetic Crystallography, Annu. Rev. Mater. Res. 45, 217 (2015).

[42] J. Rodríguez-Carvajal, Recent Advances in Magnetic Structure Determination by Neutron Powder Diffraction, Physica (Amsterdam) 192B, 55 (1993).

[43] P. Hohenberg and W. Kohn, Inhomogeneous Electron Gas, Phys. Rev. 136, B864 (1964).

[44] W. Kohn and L. J. Sham, Self-Consistent Equations Including Exchange and Correlation Effects, Phys. Rev. 140, A1133 (1965).

[45] J. P. Perdew, K. Burke, and M. Ernzerhof, Generalized Gradient Approximation Made Simple, Phys. Rev. Lett. 77, 3865 (1996).

[46] G. Kresse and J. Furthmüller, Efficient Iterative Schemes for Ab Initio Total-Energy Calculations Using a Plane-Wave Basis Set, Phys. Rev. B 54, 11169 (1996).

[47] G. Kresse and J. Furthmüller, Efficiency of Ab-Initio Total Energy Calculations for Metals and Semiconductors Using a Plane-Wave Basis Set, Comput. Mater. Sci. 6, 15 (1996).

[48] S. L. Dudarev, G. A. Botton, S. Y. Savrasov, C. J. Humphreys, and A. P. Sutton, Electron-Energy-Loss Spectra and the Structural Stability of Nickel Oxide: An LSDA + U Study, Phys. Rev. B 57, 1505 (1998).

[49] H. J. Monkhorst and J. D. Pack, Special Points for BrillouinZone Integrations, Phys. Rev. B 13, 5188 (1976). 
[50] J. P. Perdew, A. Ruzsinszky, G. I. Csonka, O. A. Vydrov, G. E. Scuseria, L. A. Constantin, X. Zhou, and K. Burke, Restoring the Density-Gradient Expansion for Exchange in Solids and Surfaces, Phys. Rev. Lett. 100, 136406 (2008).

[51] L. Zhou, Z. Tan, D. Yan, Z. Fang, Y. Shi, and H. Weng, Topological Phase Transition in Layered Magnetic Compound $\mathrm{MnSb}_{2} \mathrm{Te}_{4}$ : Spin-Orbit Coupling and Interlayer Coupling Dependence, Phys. Rev. B 102, 085114 (2020).

[52] B. Chen, F. Fei, D. Zhang, B. Zhang, W. Liu, S. Zhang, P. Wang, B. Wei, Y. Zhang, Z. Zuo et al., Intrinsic Magnetic Topological Insulator Phases in the $\mathrm{Sb}$ Doped $\mathrm{MnBi}_{2} \mathrm{Te}_{4}$ Bulks and Thin Flakes, Nat. Commun. 10, 1 (2019).

[53] L.-L. Wang, N. H. Jo, B. Kuthanazhi, Y. Wu, R. J. McQueeney, A. Kaminski, and P. C. Canfield, Single Pair of Weyl Fermions in the Half-Metallic Semimetal $\mathrm{EuCd}_{2} \mathrm{As}_{2}$, Phys. Rev. B 99, 245147 (2019).

[54] N. H. Jo, B. Kuthanazhi, Y. Wu, E. Timmons, T.-H. Kim, L. Zhou, L.-L. Wang, B. G. Ueland, A. Palasyuk, D. H. Ryan et al., Manipulating Magnetism in the Topological Semimetal EuCd ${ }_{2} \mathrm{As}_{2}$, Phys. Rev. B 101, 140402(R) (2020).

[55] R. S. K. Mong, A. M. Essin, and J.E. Moore, Antiferromagnetic Topological Insulators, Phys. Rev. B 81, 245209 (2010).

[56] S. Wimmer, J. Sánchez-Barriga, P. Küppers, A. Ney, E. Schierle, F. Freyse, O. Caha, J. Michalicka, M. Liebmann,
D. Primetzhofer et al., Ferromagnetic $\mathrm{MnSb}_{2} \mathrm{Te}_{4}$ : A Topological Insulator with Magnetic Gap Closing at High Curie Temperatures of 45-50 K, arXiv:2011.07052.

[57] L. Ding, C. Hu, F. Ye, E. Feng, N. Ni, and H. Cao, Crystal and Magnetic Structures of Magnetic Topological Insulators $\mathrm{MnBi}_{2} \mathrm{Te}_{4}$ and $\mathrm{MnBi}_{4} \mathrm{Te}_{7}$, Phys. Rev. B 101, 020412(R) (2020).

[58] Y. Deng, Y. Yu, M. Z. Shi, Z. Guo, Z. Xu, J. Wang, X. H. Chen, and Y. Zhang, Quantum Anomalous Hall Effect in Intrinsic Magnetic Topological Insulator $\mathrm{MnBi}_{2} \mathrm{Te}_{4}$, Science 367, 895 (2020).

[59] C. Liu, Y. Wang, H. Li, Y. Wu, Y. Li, J. Li, K. He, Y. Xu, J. Zhang, and Y. Wang, Robust Axion Insulator and Chern Insulator Phases in a Two-Dimensional Antiferromagnetic Topological Insulator, Nat. Mater. 19, 522 (2020).

[60] D. Ovchinnikov, X. Huang, Z. Lin, Z. Fei, J. Cai, T. Song, M. He, Q. Jiang, C. Wang, H. Li et al., Intertwined Topological and Magnetic Orders in Atomically Thin Chern Insulator $\mathrm{MnBi}_{2} \mathrm{Te}_{4}$, Nano Lett. 21, 2544 (2021).

[61] J. Ge, Y. Liu, J. Li, H. Li, T. Luo, Y. Wu, Y. Xu, and J. Wang, High-Chern-Number and High-Temperature Quantum Hall Effect without Landau Levels, Natl. Sci. Rev. 7, 1280 (2020). 